\title{
Calculation of Nucleon Electric Dipole Moments Induced by Quark Chromo-Electric Dipole Moments
}

\author{
Taku Izubuchi $^{\star a}$, Michael Abramczyk $^{b}$, Tom Blum ${ }^{a, b}$, Hiroshi Ohki $^{a}$, \\ Sergey Syritsyn ${ }^{a, c, d}$ \\ ${ }^{a}$ RIKEN/BNL Research Center, Brookhaven National Laboratory, Upton, NY 11973, USA \\ ${ }^{b}$ Physics Department, University of Connecticut, Storrs, CT 06269, USA \\ ${ }^{c}$ Department of Physics and Astronomy, Stony Brook University, Stony Brook, NY 11794, USA \\ ${ }^{d}$ Thomas Jefferson National Accelerator Facility, 12000 Jefferson Avenue, Newport News, VA \\ 23606, USA E-mail: izubuchi@quark •phy .bn l.gov
}

\begin{abstract}
We present initial results of computing nucleon electric dipole moment induced by quark chromoEDM, CP-violating quark-gluon coupling. Using chirally-symmetric domain wall and Möbius fermions with pion mass $m_{\pi}=172 \mathrm{MeV}$, we calculate the connected part of the electric dipole form factor $F_{3}\left(Q^{2}\right)$. In addition, we perform an exploratory study of the method to calculate EDM using uniform background electric field on a lattice introduced without breaking the periodicity in the time direction.
\end{abstract}

34th annual International Symposium on Lattice Field Theory

24-30 July 2016

University of Southampton, UK

* Speaker. 


\section{Introduction}

The origin of nuclear matter, or the excess of nucleons over antinucleons in the early Universe, is one of the greatest puzzles in physics known as the baryonic asymmetry of the Universe (BAU). One of the required conditions for the BAU is violation of the $C P$ symmetry. Standard Model (SM) $C P$ from CKM matrix phases is not sufficient to explain BAU, and signs of additional $C P$ are actively sought in experiments. The most promising ways to look for $C P$ are measurements of electric dipole moments (EDM) of atoms, nucleons and nuclei. Planned EDM experiments will improve their bounds by 2 orders of magnitude. Knowledge of nucleon structure and interactions is required to interpret these experiments in terms of quark and gluon fields and put constraints on many potential extensions of particle physics beyond the Standard Model (BSM) such as SUSY and GUT as sources of additional $C P$. Connecting the quark- and hadron-level effective $C P$ interactions is a task for lattice QCD (an extensive review of EDM phenomenology can be found in Ref. [1]).

Proton and neutron electric dipole moments may be induced by CP operators. The only dim4 operator is the QCD $\bar{\theta}$-angle. The $\bar{\theta}$-induced nucleon EDMs have been calculated on a lattice previously from energy shifts in background electric field [2, 3, 4] or extracting CP electric dipole form factor (EDFF) $F_{3}\left(Q^{2}\right)[5,6,7,8,9]$. Nucleon EDMs (nEDMs) induced by dim-6 operators have been studied using QCD sum rules and chiral perturbation theory [1]. Another important dim-6 operator is quark chromo-electric dipole moment (cEDM)

$$
\mathscr{L}_{c E D M}=i \sum_{\psi=u, d} \frac{\tilde{\delta}_{\psi}}{2} \bar{\psi}\left(T^{a} G_{\mu v}^{q}\right) \sigma^{\mu v} \gamma_{5} \psi,
$$

and calculations of cEDM-induced nEDMs have started using Wilson fermions [10].

In this work, we present initial calculations of nEDM induced by quark chromo-EDM using chirally-symmetric Domain wall fermions. We compute connected parts of the EDFF $F_{3}$ from 4point correlation functions of nucleon with quark current and cEDM quark-bilinear operator (1.1). In addition, we test the method to compute EDM from energy shift in background electric field, which is introduced without violating boundary conditions following Ref. [11], on a small $16^{3} \times 32$ lattice and find ballpark agreement between the two methods ${ }^{1}$.

\section{Lattice details}

In our preliminary calculation, we employ two lattice ensembles with $N_{f}=2+1$ dynamical domain wall fermions listed in Tab. 1. The first is a DSDR ensemble [12] with light pion mass $m_{\pi} \approx 172 \mathrm{MeV}$, on which we calculate the electric dipole form factor (EDFF) $F_{3}\left(Q^{2}\right)$ from vector current matrix elements of the nucleon in $C P$ vacuum. The forward limit of the EDFF gives the $\mathrm{EDM}, d_{N}=F_{3}(0) /\left(2 m_{N}\right)$. The second is a Iwasaki+DWF ensemble with heavier pion mass $m_{\pi} \approx$ $420 \mathrm{MeV}$ [13], on which we calculate the nucleon EDFF from vector current matrix elements and the nucleon EDM from nucleon energy shifts in background electric field. In large-statistics calculations on the second ensemble, we aim to compare the EDFF and EDM computed with the two different methods.

\footnotetext{
${ }^{1}$ Preliminary results presented in the conference poster did not show the form factor dependence on momentum transfer and energy shift method. We omit gradient flow study from this manuscript because of space considerations.
} 
Table 1: Lattice ensembles on which the simulations were performed. The statistics are shown for "sloppy" (low-precision) samples.

\begin{tabular}{ll|cccc|rrrr}
\hline \hline & $L_{x} \times L_{t} \times L_{5}$ & $a[\mathrm{fm}]$ & $a m_{l}$ & $a m_{s}$ & $m_{\pi}[\mathrm{MeV}]$ & conf & stat & $N_{e v}$ & $N_{C G}$ \\
\hline DSDR & $32^{3} \times 64 \times 32$ & $0.1432(7)$ & 0.001 & 0.045 & $172.3(9)$ & 28 & 896 & 1400 & 125 \\
Iwasaki & $16^{3} \times 32 \times 16$ & $0.114(2)$ & 0.01 & 0.032 & $422(7)$ & 500 & 16500 & 150 & 100 \\
\hline \hline
\end{tabular}

We use all-mode-averaging [14] framework to optimize sampling, in which we approximate quark propagators with truncated-CG solutions to a Möbius operator [15]. We use the Möbius operator with short 5 th dimension $L_{5 s}$ and complex $s$-dependent coefficients $b_{s}+c_{s}=\omega_{s}^{-1}$ (later referred to as "zMobius") that approximates the same $4 \mathrm{~d}$ effective operator as the Shamir operator with the full $L_{5 f}=32$ (DSDR) or $L_{5 f}=16$ (Iwasaki). The approximation is based on the domain wall-overlap equivalence

$$
\begin{gathered}
{\left[\not D^{\mathrm{DWF}}\right]_{4 d}=\frac{1+m_{q}}{2}-\frac{1-m_{q}}{2} \gamma_{5} \varepsilon_{L_{5}}\left(H_{T}\right), \quad H_{T}=\gamma_{5} \frac{\not D_{W}}{2+\not D_{W}},} \\
\varepsilon_{L_{5 s}}^{\text {Möbius }}(x)=\frac{\prod_{s}^{L_{5 s}}\left(1+\omega_{s}^{-1} x\right)-\prod_{s}^{L_{5 s}}\left(1-\omega_{s}^{-1} x\right)}{\prod_{s}^{L_{5 s}}\left(1+\omega_{s}^{-1} x\right)+\prod_{s}^{L_{5 s}}\left(1-\omega_{s}^{-1} x\right)} \approx \varepsilon_{L_{5 f}}^{\text {Shamir }}(x) .
\end{gathered}
$$

where the coefficients $\omega_{s}$ are chosen so that the function $\varepsilon_{L_{5}}^{\text {Möbius }}(x)$ is the minmax approximation to the $\varepsilon_{L_{5 f}}^{\text {Shamir }}(x)$. We find that $L_{5 s}=10$ is enough for efficient $4 \mathrm{~d}$ operator approximation. Shortened 5th dimension reduces the CPU and memory requirements, which is especially significant for the lighter pion mass ensemble $L_{5 f}=32$ is reduced to $L_{5 s}=10$ saving $70 \%$ of the cost. We deflate the low-lying eigenmodes of the the internal even-odd preconditioned operator, to make truncated-CG AMA efficient. The numbers of deflation eigenvectors $N_{e v}$ and truncated CG iterations $N_{C G}$ are given in Tab. 1. Figure 1 (left) shows the deviation of truncated solutions to the zMöbius operator from the exact Shamir solution, demonstrating the effect of deflating the eigenmodes between the light and the strange quark masses (Fig. 1, right). We compute 32 sloppy samples per configuration. To correct any potential bias due to the approximate $\not D$ operator and truncated CG, we compute one exact sample with the Shamir operator per configuration. The latter is computed iteratively by refining the solution of the "zMobius" to approach the solution of the Shamir operator, again taking advantage of the short $L_{5 s}$ and deflation.

\section{Electric Dipole Form factor $F_{3}$}

The EDFF $F_{3}$ is a parity-odd quantity induced by $C P$ interactions. To compute the effect of chromo-EDM (1.1), we Taylor-expand QCD $+C P$ vacuum averages as $S \rightarrow S+i \sum_{x} \tilde{\delta}_{\psi} \mathscr{O}_{\psi, x}^{\overline{C P}}$

$$
\left\langle N\left[\bar{q} \gamma_{\mu} q\right] \bar{N}\right\rangle_{C P}=C_{N J \bar{N}}-i \tilde{\delta}_{\psi} \delta_{\psi}^{\overline{C P}} C_{N J \bar{N}}+O\left(\tilde{\delta}_{\psi}^{2}\right),
$$

with $\quad C_{N J \bar{N}}=\left\langle N\left[\bar{q} \gamma_{\mu} q\right] \bar{N}\right\rangle, \quad \delta_{\psi}^{\overline{C P}} C_{N J \bar{N}}=\left\langle N\left[\bar{q} \gamma_{\mu} q\right] \bar{N} \cdot \sum_{x} \mathscr{O}_{\psi, x}^{\overline{C P}}\right\rangle, \quad \mathscr{O}_{\psi}^{\overline{C P}}=\frac{1}{2} \bar{\psi}\left(T^{a} G_{\mu \nu}^{a}\right) \sigma^{\mu v} \gamma_{5} \psi$,

where $\langle\cdot\rangle$ in the second line stand for vacuum averages computed with $C P$-even action $S$. In this work, we calculate only fully connected contributions to the correlation functions $C_{N J \bar{N}}$ and 

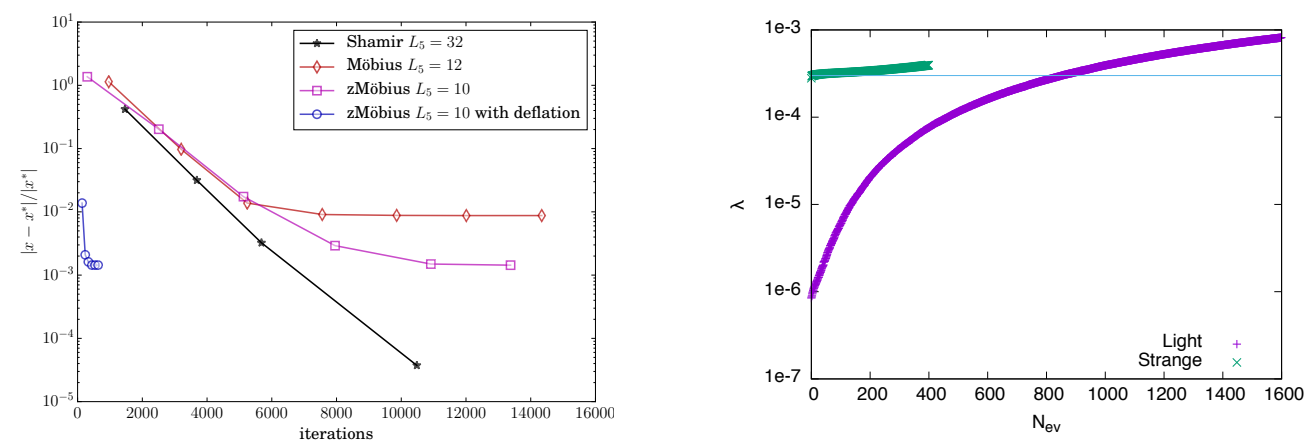

Figure 1: (Left) Comparison of $4 \mathrm{~d}$ solution vectors (quark propagators) between approximate 5D Dirac operators ( $L_{5}=10$ complex "zMobius" and $L_{5}=12$ regular Mobius) and the exact Shamir quark propagator with $L_{5}=32$ vs the iteration count. (Right) Low-mode eigenvalues of zMobius $L_{5}=10$ computed with light and strange quark masses.
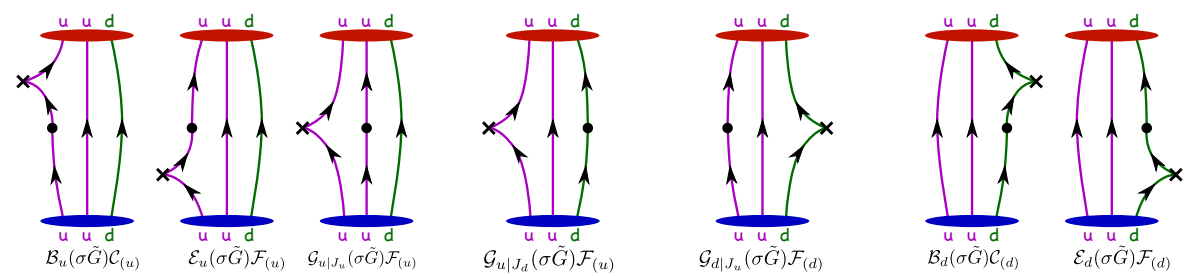

Figure 2: Quark-connected contractions of nucleon, quark current, and cEDM operators.

$\delta^{\overline{C P}} C_{N J \bar{N}}$. To compute the latter, the quark-bilinear cEDM density (1.1) is inserted once in every $\psi$-quark line of $C_{N J \bar{N}}$ diagrams, generating the four-point functions shown in Fig.2. We evaluate these 4-point contractions using sequential propagators. In addition to the usual one forward and two backward (sink-sequential) propagators, we compute one cEDM-sequential and four doublysequential ( $\{\mathrm{cEDM}$, sink $\}$-sequential) propagators. This is the minimum number inversions required to compute cEDM-induced nEDM for all combinations of two degenerate flavors $u$ and $d$, with one source-sink separation $t_{\text {sep }}$ and one sink momentum $\vec{p}^{\prime}$. Compared to the method used in Ref. [10], in effect we compute the first derivative in the $C P$-odd coupling $\varepsilon$ at $\varepsilon=0$, obviating any higher-order dependence on $\varepsilon$. We use $\vec{p}^{\prime}=0$ and $t_{\text {sep }}=8 a=1.14 \mathrm{fm}$ for the $32^{3} \times 64$ and $t_{\text {sep }}=\{8,10\} a=0.91,1.15 \mathrm{fm}$ for the $16^{3} \times 32$ ensemble. The $C P$-even part of the nucleon-current correlator (3.1) requires only contractions but no additional inversions. Both $C_{N J \bar{N}}$ and $\delta^{\overline{C P}} C_{N J \bar{N}}$ are computed with the polarization projector $T_{\mathrm{pol}}=\frac{1+\gamma_{4}}{2} \Sigma_{3}=\frac{1+\gamma_{4}}{2}\left(-i \gamma_{1} \gamma_{2}\right)$. This projector is sufficient to extract form factors $F_{1,2}$ and $F_{3}$ from $C P$ nucleon matrix elements of the vector current ${ }^{2}$

$$
\left\langle N_{p^{\prime}}\left|\bar{q} \gamma^{\mu} q\right| N_{p}\right\rangle=\bar{u}_{p^{\prime}}\left[F_{1}\left(Q^{2}\right) \gamma^{\mu}+F_{2}\left(Q^{2}\right) \frac{\sigma^{\mu v} q_{v}}{2 m_{N}}+F_{3}\left(Q^{2}\right) \frac{i \gamma_{5} \sigma^{\mu v} q_{v}}{2 m_{N}}\right] u_{p} .
$$

We employ the usual $C_{3 p t} / C_{2 p t}$ ratios to cancel overlaps and exponential factors and we extract matrix elements of the ground state as the central plateau average (see Fig. 4, right). Excited state contaminations are neglected in this preliminary analysis. The P-even form factors $F_{1,2}$ are

\footnotetext{
${ }^{2}$ All conventions for form factors and momenta are Euclidean
} 

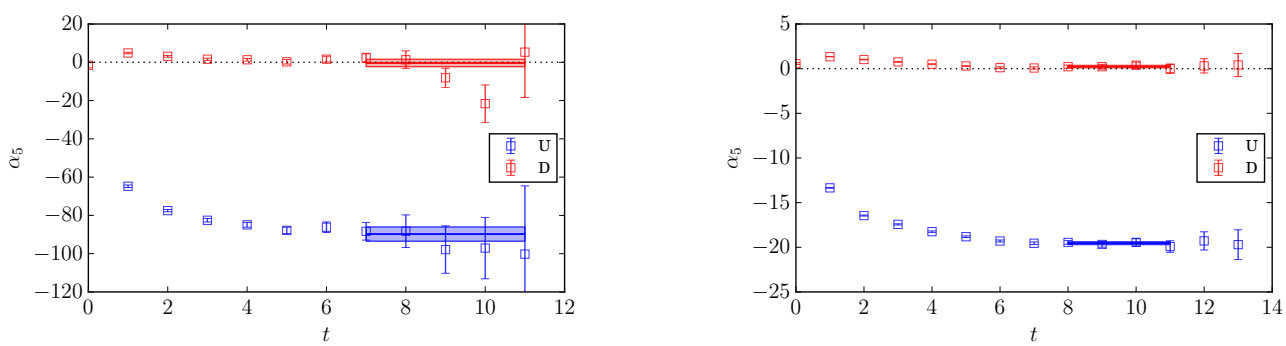

Figure 3: The parity-mixing phase of the proton propagator induced by $u$ - and $d$-cEDM, for the $m_{\pi}=172$ (left) and $422 \mathrm{MeV}$ (right). The mixing is noticeably larger when the unpaired quark is affected by cEDM.
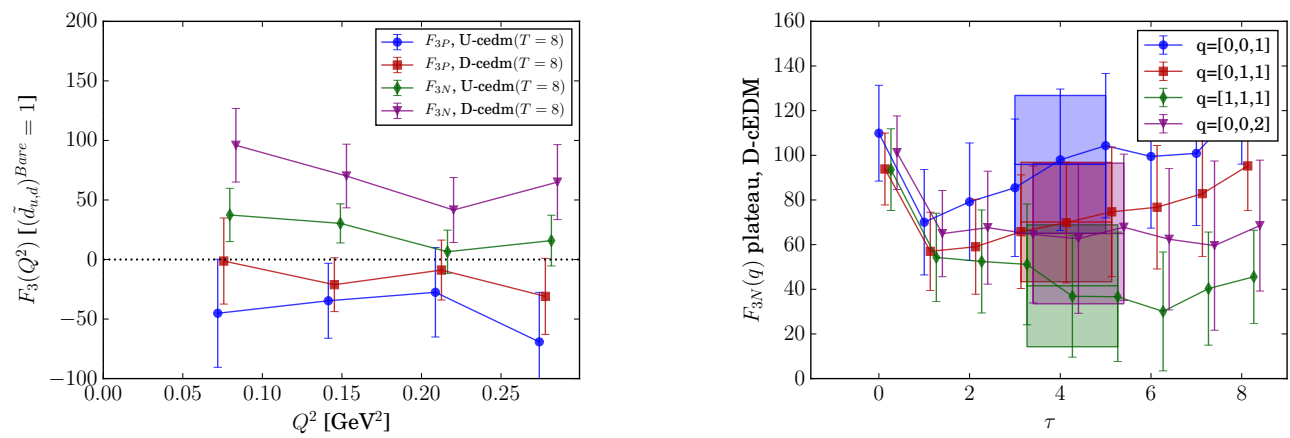

Figure 4: Form factors computed on $m_{\pi}=172$ ensemble: proton and neutron $\operatorname{EDFF} F_{3}\left(Q^{2}\right)$ induced by $u$ and $d$-cEDM (left) and form factor plateaus for neutron $F_{3}$ induced by $d$-quark cEDM (right). Source-sink separation is $t_{\mathrm{sep}}=8 a=1.15 \mathrm{fm}$.

extracted from overdetermined fit (e.g., Ref. [16]) of $C_{N^{\mu}{ }_{N}}$ combining all orientations of the current and momenta. The EDFF $F_{3}$ is extracted from the timelike-component of $\delta^{\overline{C P}} C_{N J^{4} \bar{N}}$. CP interaction leads to parity mixing of the nucleon spinors

$$
\tilde{u}_{p}=e^{i \alpha_{5} \gamma_{5}} u_{p}, \quad\left\langle N_{\delta}(t, \vec{p}) \bar{N}_{\delta^{\prime}}(0)\right\rangle \stackrel{t \rightarrow \infty}{=} \frac{\left[-i \not p+m_{N} e^{2 i \alpha_{5} \gamma_{5}}\right] \delta \delta^{\prime}}{2 E_{N}} e^{-E_{N} t}
$$

that complicates analysis of $F_{3}$, as $\delta^{\overline{C P}} C_{N J^{4} \bar{N}}$ receives contributions from $F_{1,2}$ due to "polarization mixing" $\sim \alpha_{5}\left\{\gamma_{5}, T_{\mathrm{pol}}\right\}$ [17]. To subtract them, we determine the cEDM flavor-dependent mixing angle from the $C P$-odd two-point function $\delta^{\overline{C P}} C_{N \bar{N}}$ as (see Fig. 3)

$$
\alpha_{5}(t)=-\operatorname{Re} \operatorname{Tr}\left[T^{+} \gamma_{5} \delta^{\overline{C P}} C_{N \bar{N}}(t)\right] / \operatorname{Re} \operatorname{Tr}\left[T^{+} C_{N \bar{N}}(t)\right],
$$

where $T^{+}=\left(1+\gamma_{4}\right) / 2$ is the positive-parity projector. Remarkably, connected cEDM leads to parity rotation only when $C P$ interaction affects the "spectator" (non-diquark) flavor in the nucleon. We use the value $\alpha_{5}\left(t_{\mathrm{sep}}\right)$ and $\tau$-dependent plateau values of $F_{1,2}$ for the subtraction. The subtracted formula for $F_{3}$ is [17]

$$
F_{3}^{\psi}=\frac{2 m_{N}}{E_{N}+m_{N}}\left[\frac{2 E_{N}\left(E_{N}+m_{N}\right)}{q_{3}} \operatorname{Tr}\left[T^{+} \Sigma_{z} \delta_{\psi}^{\overline{C P}} R_{N J^{4} \bar{N}}\right]-\alpha_{5} G_{E}\right],
$$

where spin $\Sigma_{z}=-i \gamma_{1} \gamma_{2}$ and $\delta^{\overline{C P}} R_{N J^{4} \bar{N}}$ is the $\delta^{\overline{C P}} C_{3 p t} / C_{2 p t}$ ratio canceling nucleon field normalization and time exponentials. The $F_{3}$ results for the $m_{\pi}=172 \mathrm{MeV}$ ensemble are shown in Fig. $4^{3}$

\footnotetext{
${ }^{3}$ On one out of 30 analyzed configurations, we have encountered an outlier sample that had values for the 2- and 3-point functions strongly deviating from the rest of the ensemble. We have omitted the entire gauge configuraion as well as one adjacent to it from the preliminary analysis, and we are investigating what caused this outlier.
} 


\section{Energy shift in background electric field}

The background field method provides opportunity to compute EDMs directly, whereas $Q^{2} \rightarrow$ 0 extrapolation of $F_{3}\left(Q^{2}\right)$ may lead to systematic uncertainties. We have done an exploratory calculation of cEDM-induced nEDM on the $16^{3} \times 32 m_{\pi}=422 \mathrm{MeV}$ ensemble in uniform electric field to compare these two methods. We use the Wick-rotated electric field, which, if quantized as $Q_{q} \mathscr{E}_{k}=n_{k} \frac{2 \pi}{L_{k} L_{t}}$, does not break (a)periodic boundary conditions [11]. The Wick-rotated electric field cannot create pion pairs out of vacuum, but it can "accelerate" charged particles and complicate calculation of their rest mass. For this reason, we apply this analysis only to the neutron.

We compute the energy shift from the $C P$-odd correction $\delta^{\overline{C P}} C_{N \bar{N}, \mathscr{E}_{z}}$ to the neutron propagator in presence of the uniform electric field $\mathscr{E}_{z}$

$$
U_{\mu} \rightarrow U_{\mu} e^{i Q A_{\mu}}, \quad A_{\hat{t}}(z, t)=z \mathscr{E}_{z}\left(\forall z=0 \ldots\left(L_{z}-1\right)\right), \quad A_{\hat{z}}(z, t)=-t L_{z} \mathscr{E}\left(\forall t=L_{t}-1\right) .
$$

$\delta^{\overline{C P}} C_{N \bar{N}, \mathscr{E}_{z}}$ is computed similarly to Fig. 2 but without the quark current. $C P$-odd interaction leads to spin-dependent energy shift $\delta E=-d_{N} \hat{S} \cdot i \overrightarrow{\mathscr{E}}$, from which we extract $d_{N}$ as

$$
2 m_{N} d_{N}^{\mathrm{eff}}(t)=-\frac{2 m_{N}}{\mathscr{E}_{z}}\left[R_{z}(t+1)-R(t)\right], \quad R_{z}(t)=\frac{\operatorname{Tr}\left[T^{+} \Sigma_{z} \delta^{\overline{C P}} C_{N \bar{N}, \mathscr{E}_{z}}(t)\right]}{\operatorname{Tr}\left[T^{+} C_{N \bar{N}, \mathscr{E}_{z}}(t)\right]} .
$$

We have computed $d_{N}^{\text {eff }}(t)$ for two values of the electric field $\left(1 \times\right.$ and $\left.2 \times \mathscr{E}_{\min }\right)$, and averaged the result over $\mathscr{E}_{z}$ and $S_{z}$ directions. The statistics are the same as in the form factor calculation.

In Figure 5 we compare $F_{3}\left(Q^{2}\right)$ and $2 m_{N} d_{N}^{\text {eff }}(t)$, where the EDM "units" are adjusted for a direct comparison. The EDM value from the energy shift appears to reach plateau for $t=4 \ldots 6$. However, the comparison EDFF values computed with $t_{\text {sep }}=8 a$ and $10 a$ shows that $F_{3}$ requires detailed analysis of excited state contributions. For the $d$-cEDM, there appears to be $\times 2 \ldots \times 2.5$ difference between $d_{N}^{\text {eff }}$ and $F_{3}\left(Q_{\text {min }}^{2}\right)$. Considering the trend of $F_{3}\left(Q^{2}\right)$ with $Q^{2} \rightarrow 0$, the results of form factor and energy shift calculations appear to be compatible. We note that the minimal electric field is $\mathscr{E}_{\min }=0.097 \mathrm{GeV}^{2}=490 \mathrm{MeV} / \mathrm{fm}$ on the small $16^{3} \times 32$ lattice. Such strong electric field may distort the nucleon and introduce systematic shift to $d_{N}^{\text {eff }}$, complicating the comparison. Nevertheless, we find the data suggest encouraging agreement between the two methods.
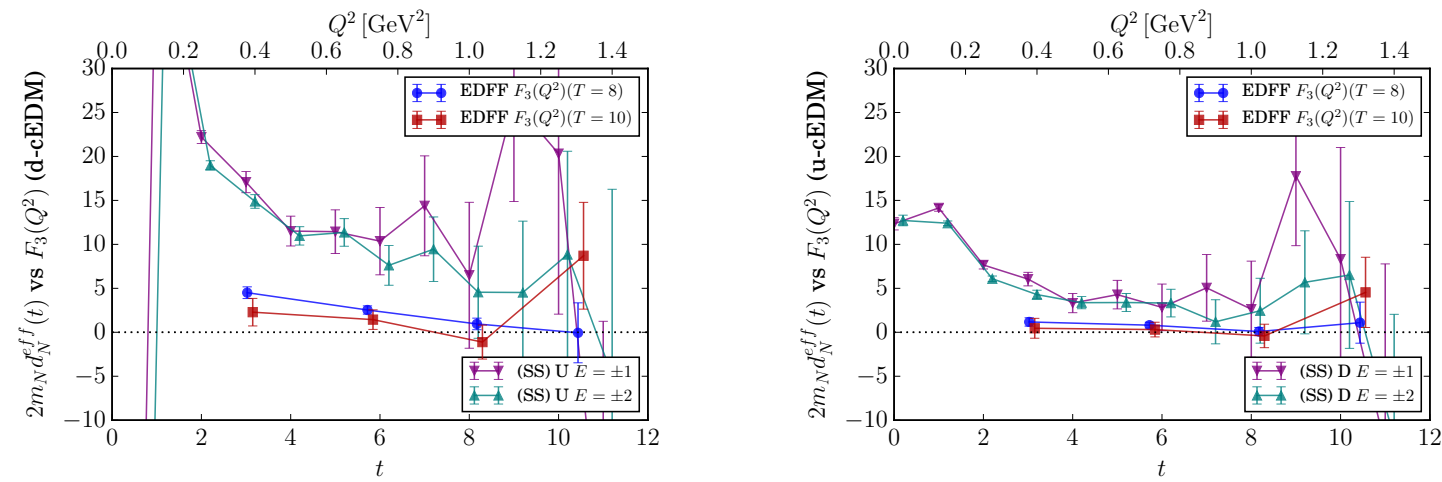

Figure 5: Comparison of neutron EDFF $F_{3}\left(Q^{2}\right)$ vs. $Q^{2}$ (top scale) effective $\operatorname{EDM} 2 m_{N} d_{N}^{\text {eff }}(t)$ (4.2) vs. $t$ (bottom scale) computed from energy shift on the $422 \mathrm{MeV}$ ensemble, for $d$-cEDM (left) and $u$-cEDM(right). 


\section{Summary}

We have obtained preliminary results for the nucleon electric dipole form factors induced by light quark chromo-EDM interaction using chirally-symmetric domain wall fermions. Our initial calculation does not take into account renormalization and mixing of chromo-EDM operators with other $C P$ operators, and neglects effects of excited states. In addition, we have performed an exploratory study of electric dipole moments using the background electric field method and have compared them to the form factor results. While the statistical and systematic precision is insufficient to draw definite conclusions, these results appear to be comparable. Using methods developed in this work, the studies will be extended to the physical point and will include analysis of excited states, as well as renormalization and mixing of $C P$ operators.

\section{ACKNOWLEDGEMENTS}

We are grateful to the RBC/UKQCD collaboration for the gauge configurations and to the RIKEN ACCC facility for the computing resources provided for this work. T.B. is supported by US DOE grant DE-FG02-92ER40716. T.I. is supported in part by the Japanese Ministry of Education Grant-in-Aid, No. 26400261 The work of H.O. is supported by the RIKEN Special Postdoctoral Researcher program. S.S. thanks RBRC for support under its joint tenure track fellowship with Stony Brook University S.S. also thanks KITP UCSB for hospitality at the "Nuclear16" workshop and support by the National Science Foundation under Grant No. NSF PHY11-25915.

\section{References}

[1] J. Engel, M. J. Ramsey-Musolf, and U. van Kolck, Prog.Part.Nucl.Phys. 71, 21 (2013).

[2] S. Aoki and A. Gocksch, Phys. Rev. Lett. 63, 1125 (1989), [Erratum: Phys. Rev. Lett.65,1172(1990)].

[3] E. Shintani et al., Phys.Rev. D75, 034507 (2007).

[4] E. Sintani, S. Aoki, and Y. Kuramashi, Phys.Rev. D78, 014503 (2008).

[5] F. Berruto, T. Blum, K. Orginos, and A. Soni, Phys.Rev. D73, 054509 (2006).

[6] E. Shintani et al., Phys.Rev. D72, 014504 (2005).

[7] A. Shindler, T. Luu, and J. de Vries, Phys. Rev. D92, 094518 (2015).

[8] E. Shintani, T. Blum, T. Izubuchi, and A. Soni, Phys. Rev. D93, 094503 (2016).

[9] C. Alexandrou et al., Phys. Rev. D93, 074503 (2016).

[10] T. Bhattacharya et al., PoS LATTICE2015, 238 (2015).

[11] W. Detmold, B. C. Tiburzi, and A. Walker-Loud, Phys. Rev. D79, 094505 (2009).

[12] R. Arthur et al., Phys. Rev. D87, 094514 (2013).

[13] T. Blum et al., Phys. Rev. D84, 114503 (2011).

[14] E. Shintani et al., Phys. Rev. D91, 114511 (2015).

[15] R. Brower, H. Neff, and K. Orginos, Nucl.Phys.Proc.Suppl. 153, 191 (2006).

[16] S. Syritsyn et al., Phys.Rev. D81, 034507 (2010).

[17] M. Abramczyk et al., (2017), arXiv:1701.07792[hep-lat]. 